\title{
Effect of folic acid on prenatal alcohol-induced modification of brain proteome in mice
}

\author{
Yajun $\mathrm{Xu}^{*}$, Yunan Tang and Yong Li \\ Department of Nutrition and Food Hygiene, School of Public Health, Peking University, Beijing 100083, China \\ (Received 19 December 2006 - Revised 5 July 2007 - Accepted 5 July 2007)
}

Maternal alcohol consumption during pregnancy can induce central nervous system abnormalities in the fetus, and folic acid supplementation can reverse some of the effects. The objective of the present study was to investigate prenatal alcohol exposure-induced fetal brain proteome alteration and the protective effect of folic acid using proteomic techniques. Alcohol $(5 \cdot 0 \mathrm{~g} / \mathrm{kg})$ was given intragastrically from gestational day (GD) 6 to 15 , with or without $60.0 \mathrm{mg}$ folic acid/kg given intragastrically during GD1-16 to pregnant Balb/c mice. The control group received distilled water only. Results of litter evaluation on GD18 showed that supplementation of folic acid reversed the prevalence of microcephaly induced by alcohol. Proteomic analysis indicated that, under the dosage of the present investigation, folic acid mainly reversed the alcohol-altered proteins involved in energy production, signal pathways and protein translation, which are all important for central nervous system development.

Alcohol: Folic acid: Brain proteome: Fetal alcohol syndrome

The toxic effect of maternal alcohol consumption during pregnancy has been documented over the last two decades since Jones \& Smith first described the characteristics of fetal alcohol syndrome (FAS) in $1973^{1}$. As a result of these studies, the relationship between maternal alcohol consumption and congenital neurological abnormalities is well established ${ }^{2-4}$. Microcephaly, defined as reduced brain weight relative to body weight, is a classic indicator of central nervous system (CNS) malformation, which is central to a diagnosis of $\mathrm{FAS}^{5}$. FAS patients often have more than one neurodevelopmental disorder, including substantial, lifelong impairments in intellectual, cognitive and psychosocial functioning ${ }^{6-9}$. The most frequent problems include attention deficit, sequential processing (short-term memory and encoding) impairment, neuropsychiatric abnormalities and hyperactivity, which might contribute to the likelihood of committing crimes ${ }^{10,11}$.

Animal models of FAS have been used successfully to examine the effect of alcohol exposure during development on social behaviour. It has been shown that prenatal alcohol exposure affects the learning and memory abilities, and the executive functioning of rodents ${ }^{3,12,13}$. The social behaviour deficits found in rodent pups following perinatal alcohol exposure were consistent with the frequently observed clinical symptoms in FAS children ${ }^{14}$.

Folic acid (FA) has been credited with a beneficial role in preventing a range of birth defects, especially neurological abnormalities ${ }^{15-18}$. Lalonde et al. ${ }^{19}$ also found that increases in the plasma level of FA decreased perseverative responding and improved spatial memory in rats. In the present investigation, we studied the effect of FA on prenatal alcohol-induced fetal brain proteome modification in mice, hoping to provide a molecular clue to the protective mechanism of FA on alcohol-related abnormalities in the CNS.

\section{Materials and methods}

\section{Chemicals}

Anhydrous ethanol was purchased from the Beijing Chemical Company (Beijing, China). FA was purchased from the Sigma Chemical Company (St Louis, MO, USA). Acrylamide, ammonium persulfate, isoelectric $\mathrm{pH}$ gradient strips $(5 \mathrm{~mm}$ wide, $18 \mathrm{~cm}$ long, $\mathrm{pH} \mathrm{3-10} \mathrm{linear),} \mathrm{urea,} \mathrm{and} \mathrm{ultrapure}$ reagents for polyacrylamide gel preparation were obtained from Bio-Rad (Hercules, CA, USA). Carrier ampholytes $(\mathrm{pH}$ 4-8) and 3-[(3-cholamidopropyl) dimethylammonio]-1-propane sulfonate (CHAPS) were also obtained from Bio-Rad. HPLC-grade methanol and glacial acetic acid were used. Dithiothreitol, $N, N, N^{\prime}, N^{\prime}$-tetramethylethylendiamine, iodoacetamide, ammonium bicarbonate, trifluoroacetic acid and $\alpha$-cyano-4-hydroxycinnamic acid were obtained from the Sigma Chemical Company. Modified porcine trypsin was purchased from Promega (Madison, WI, USA). All chemicals (unless specified) were reagent grade and used without further purification. High-purity water was prepared from a Milli-Q gradient water-purification system (Millipore, Bedford, MA, USA) and was used for proteome analysis in the present study.

Abbreviations: CNS, central nervous system; FA, folic acid; FAS, fetal alcohol syndrome; GD, gestational day; TCTP, translationally controlled tumour protein.

* Corresponding author: Dr Yajun Xu, fax +86 10 82801575, email yajun_xucn@126.com 


\section{Animals and treatment}

Virgin female Balb/c mice, age 8-12 weeks, were used in the research. The animals were maintained in a temperature- and humidity-controlled animal facility with a $16 \mathrm{~h}$ light $-8 \mathrm{~h}$ dark cycle, and were provided food and water ad libitum throughout the study. The FA content of the chow was $2.0 \mathrm{mg} / \mathrm{kg}$. Dams were caged with sires overnight and copulation was assessed the following morning by the presence of a vaginal plug to signify gestational day (GD) 0 .

Plug-positive females were randomly divided into three groups (ten dams per group). Dams in the FAS model group were administered $5.0 \mathrm{~g}$ alcohol $/ \mathrm{kg}$ body weight intragastrically as a $25 \%(\mathrm{v} / \mathrm{v})$ solution in twice-distilled water, using a 21 -gauge needle. They were treated at 08.00 hours daily from GD6 to GD15 to cover the whole organogenesis period $^{20}$. The FA supplementation group was additionally dosed daily with $60.0 \mathrm{mg}$ FA/kg from GD1 to GD15. During the alcohol treatment period, FA was given at 10.00 hours $(2 \mathrm{~h} \text { after ethanol administration })^{21,22}$. Controls received an equal volume of twice-distilled water without alcohol or FA. Food consumption of each dam was recorded every $3 \mathrm{~d}$. The use of animals in the research was approved by, and conducted in compliance with, the guidelines for animal research of Peking University.

\section{Fetal manipulations}

All dams were killed on GD18. The fetuses were delivered by rapid hysterectomy, weighed and killed by cervical dislocation. The fetal brain was removed, wiped and weighed immediately.

\section{Protein extraction}

Two fetal brains with no obvious gross malformations per litter and six litters per group were selected for protein extraction. Each fetal brain was prepared individually. The whole brain tissue was suspended in $0.5 \mathrm{ml}$ of sample buffer consisting of 7 M-urea (Merck, Darmstadt, Germany), 2 M-thiourea (Sigma, St Louis, MO, USA), $4 \%$ (w/v) 3-[(3-cholamidopropyl) dimethylammonio]-1-propane sulfonate (CHAPS; Sigma, St Louis, MO, USA), $65 \mathrm{~mm}$-1,4-dithioerythritol (Merck, Darmstadt, Germany), 0.2\% (w/v) 3/10 ampholytes (BioRad), and $10 \mu \mathrm{l}$ mixture of protease inhibitors (Roche Diagnostics, Mannheim, Germany). The suspension was sonicated for approximately $30 \mathrm{~s}$, mixed with $5 \mu \mathrm{l}$ DNAse and RNAse (Promega, Madison, WI, USA), left at room temperature for $1 \mathrm{~h}$ and then centrifuged at $14000 \mathrm{~g}$ for $60 \mathrm{~min}$. The protein content in the supernatant fraction was determined by the Coomassie blue method ${ }^{23}$.

\section{Two-dimensional electrophoresis}

Samples of $1 \mathrm{mg}$ protein were applied on immobilised isoelectric point 3-10 non-linear gradient strips in sample cups at their basic and acidic ends. Isoelectric $\mathrm{pH}$ gradient strips were rehydrated for $12 \mathrm{~h}$ at $30 \mathrm{~V}$ and $20^{\circ} \mathrm{C}$ using the IPGphor (Amersham Biosciences, Barcelona, Spain). The voltage during isoelectric focusing (IEF) at $20^{\circ} \mathrm{C}$ was programmed as follows: $1 \mathrm{~h}$ at $500 \mathrm{~V}, 1 \mathrm{~h}$ at $1000 \mathrm{~V}, 30 \mathrm{~min}$ at
$8000 \mathrm{~V}$ (gradient), and $8 \mathrm{~h}$ at $8000 \mathrm{~V}$. Immediately after IEF separation, the strips were equilibrated in SDS equilibration solution (50 mMc-tri(hydroxymethyl)-aminomethane- $\mathrm{HCl}(\mathrm{pH}$ 8.8), 6 M-urea, $30 \%$ glycerol, $2 \%$ SDS and bromophenol blue (trace)). In the first equilibration step $1 \%$ dithiothreitol was added to the solution. In the second equilibration step, $4 \%$ iodoacetamide was added. The second-dimensional separation was performed on $13 \%$ gradient sodium dodecyl sulfate polyacrylamide gels. The gels were run at $40 \mathrm{~mA}$ per gel for $40 \mathrm{~min}$ and $60 \mathrm{~mA}$ for another $5 \mathrm{~h}$. After protein fixation for $12 \mathrm{~h}$ in $40 \%$ methanol and $10 \%$ acetic acid, the gels were stained with colloidal Coomassie blue (Novex, San Diego, CA, USA) for $8 \mathrm{~h}$. Molecular masses were determined by running standard protein markers (Pharmacia, Uppsala, Sweden), covering the range $10-200 \mathrm{kDa}$. Isoelectric point values were used as given by the supplier of the immobilised $\mathrm{pH}$ gradient strips. Excess dye was washed out from the gels with distilled water. All the gels were scanned with a PowerLook 1000 Scanner (Bio-Rad) and the images were saved as TIFF files for further analysis.

\section{Image analysis}

Image analysis and quantification of spots were performed with PDQuest 7.0 software (Bio-Rad). Spot detection was optimised by adjusting the default parameters. Matching of the spots was performed by choosing a gel as a reference gel, manually selecting twenty to fifty spots as user seed, depending on the gel pattern, and matching all of the spots. Additional matching was performed manually when it was necessary. Spot standardisation was carried out for all matched spots. The background was subtracted manually by selecting approximately sixty-five non-spot areas. Spot intensities were then normalised as percentage of the total spot intensity using spots that were present in all gels. The spot intensity data of each sample were exported to Microsoft Excel (Redmond, WA, USA). Since two samples per litter and six litters per group were applied to two-dimensional electrophoresis and image analysis, altogether twelve datasheets per group were obtained. Then the average intensity of each matched spot was calculated and the intensity difference of each matched spot between different groups was found.

\section{In-gel digestion of protein}

Protein spots found to have significantly altered levels of expression were excised from the gels, and washed with ion-free water and $50 \%$ acetonitrile in $100 \mathrm{~mm}$-ammonium bicarbonate. After washing, gel pieces were shrunk by the addition of acetonitrile and dried. The dried gel pieces were rehydrated with trypsin $(40 \mathrm{ng} / \mu \mathrm{l})$ (Roche Diagnostics) in enzyme buffer (consisting of $5 \mathrm{~mm}$-octylb-d-glucopyranoside and $10 \mathrm{mM}$-ammonium bicarbonate) and incubated for $4 \mathrm{~h}$ at $30^{\circ} \mathrm{C}$. Peptide extraction was performed with $10 \mathrm{ml} 1 \%$ trifluoroacetic acid in $5 \mathrm{~mm}$-octylb-D-glucopyranoside. Extracted peptides were directly applied onto a target (MTP AnchorChipTM 600/384; Bruker Daltonics, Bremen, Germany) that was loaded with a thin-layer matrix of $\alpha$-cyano-4-hydroxycinnamic acid (Sigma, St Louis, MO, USA). 


\section{Mass spectroscopy}

A small fraction $(0 \cdot 5 \mu \mathrm{l})$ of the unseparated tryptic digest mixture was mixed with $\alpha$-cyano-4-hydroxycinnamic acid matrix $(1: 1, v / v)$ and analysed on an Ultraflex ${ }^{\mathrm{TM}}$ TOF/TOF (Bruker Daltonics) matrix-assisted laser desorption-ionisation time of flight MS.

\section{Database searches for protein identification}

The peptide masses were entered into the MASCOT search engine (www.matrixscience.com). The following parameters were used in the searches: Mus musculus taxonomy, trypsin digest, up to one missed cleavage allowed, monoisotopic masses and a mass tolerance of 100 parts per million. The National Center for Biotechnology Information (NCBI) and Swiss-Prot databases were searched to match the tryptic peptide fingerprint with a parent polypeptide.

\section{Statistical analysis}

All dataset analyses were performed using the Statistical Package for Social Sciences for Windows (version 13.0; SPSS Inc., Chicago, IL, USA).

The body and brain weights of each fetus in one litter were recorded, and the averages of body and brain weights of this litter were calculated. The same calculating method was used for other litters in the same group, and then the average of averages was calculated as the mean for this group. Intergroup differences were analysed using ANOVA followed by the least significant difference post hoc test. The level of significance was set at $P<0.05$.

For the protein spots' intensity analysis, the intensity of each matched spot was analysed separately. The average intensity of each matched spot for one group was calculated from the twelve data in this group. Then inter-group differences were analysed using ANOVA, followed by the least significant difference post hoc test. The level of significance was set at $P<0 \cdot 05$.

\section{Results}

\section{Food consumption}

During the whole experiment, the average food consumption of the dams in the control, FAS model and FA supplementation groups was 121.83 (SD 18.16), 111.67 (SD 14.67) and
121.25 (SD 11.88) g, respectively. No significant difference of food consumption between the three groups was found $(P>0 \cdot 05)$.

\section{Gross examination of the fetuses}

Four fetuses with exencephaly malformation were found in the FAS model group with none found in the control and FA-supplemented groups. Statistical analysis showed significant decreases of fetal body weight and brain weight in the FAS model group, suggesting in utero growth retardation. The fetal brain:body weight ratio also decreased in the FAS model group, which is characteristic in FAS, defined as 'microencephaly'. However, the ratio was reversed by FA supplementation (Table 1).

\section{Expression profile of fetal brain proteins}

Intact fetal whole-brain protein samples from the control, FAS model and FA-supplemented groups were analysed individually. Image analysis revealed that the abundances of over thirty individual protein spots were significantly changed in the FAS model group compared with those of the control group; seventeen of these were reversed by FA supplementation. The seventeen protein spots were gently cut and subjected to tryptic digestion and matrix-assisted laser desorption-ionisation time of flight MS analysis. As a result, thirteen spots were matched to known protein sequences and identified; the remaining four spots could not be identified, as generated peptide fragments did not match with mouse sequences in public databases or were too low in abundance to obtain useful data. Of the thirteen identified protein spots, ten were increased, and three were decreased as a consequence of in utero alcohol exposure (Fig. 1). FA supplementation reversed the expression changes of all the thirteen proteins, however, to different degrees, as indicated by the mean abundance changes of protein spots in Table 2. Proteins identified mainly include enzymes involved in metabolism and energy production, protein translation, folding and signalling (Table 2).

\section{Discussion}

Prenatal alcohol exposure-induced CNS abnormalities have a far-reaching impact on human life. CNS dysfunctions, such as impaired neural plasticity, poor learning and memory

Table 1. Effect of folic acid (FA) on prenatal ethanol-induced gross malformation of mice fetuses

(Mean values and standard deviations)

\begin{tabular}{|c|c|c|c|c|c|c|c|c|c|}
\hline \multirow[b]{2}{*}{ Group } & \multirow[b]{2}{*}{ Litters $(n)$} & \multirow[b]{2}{*}{ Fetuses $(n)$} & \multirow[b]{2}{*}{ Exencephaly $(n)$} & \multicolumn{2}{|c|}{ Brain weight $(\mathrm{mg}) \ddagger$} & \multicolumn{2}{|c|}{$\begin{array}{l}\text { Body weight } \\
\text { (g) } \ddagger\end{array}$} & \multicolumn{2}{|c|}{$\begin{array}{c}\text { Brain:body } \\
\text { weight }(\%) \ddagger\end{array}$} \\
\hline & & & & Mean & SD & Mean & SD & Mean & SD \\
\hline Control & 10 & 89 & 0 & $447 \cdot 71$ & $16 \cdot 91$ & $1 \cdot 38$ & 0.14 & 32.44 & $4 \cdot 24$ \\
\hline FAS model & 10 & 74 & 4 & $398 \cdot 83^{\star \star}$ & 28.08 & $1 \cdot 30^{\star *}$ & 0.12 & $30 \cdot 89^{\star \star}$ & $3 \cdot 86$ \\
\hline Ethanol + FA & 10 & 83 & 0 & $427 \cdot 90^{\star \star}+\dagger$ & $19 \cdot 05$ & $1.34^{*} \dagger$ & 0.09 & 31.93† & 3.32 \\
\hline
\end{tabular}

FAS, fetal alcohol syndrome.

Mean value was significantly different from that of the control group: ${ }^{\star} P<0.05,{ }^{\star *} P<0.001$.

Mean value was significantly different from that of the FAS model group: $\uparrow P<0.05, \dagger \dagger P<0.001$.

‡Fetuses with exencephaly were excluded. 
pl3

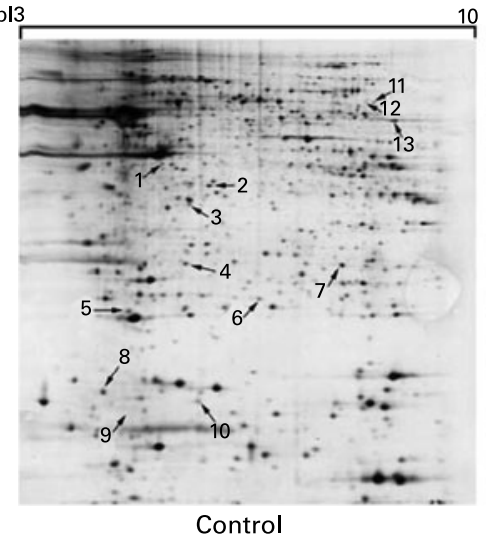

103

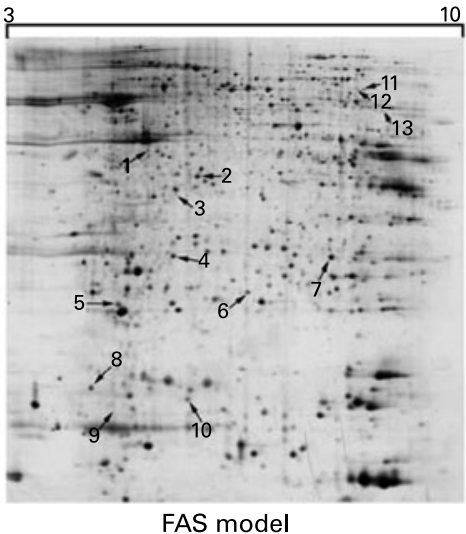

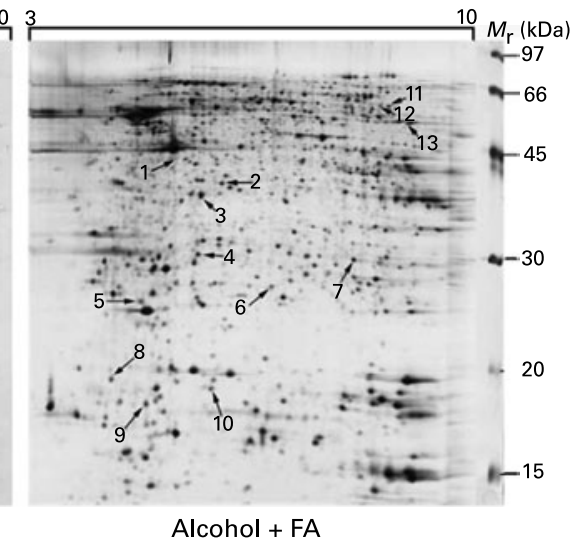

Fig. 1. Two-dimensional gel electrophoresis of whole fetal brains from control, fetal alcohol syndrome (FAS) model and folic acid (FA)-supplemented mice. The identified proteins are indicated by arrows and numbered. The protein names are listed in Table 2. pl, isoelectric point; $M_{\mathrm{r}}$, molecular mass.

capabilities, neuropsychiatric abnormalities and hyperactivity, are common in individuals with prenatal alcohol exposure $^{24}$. In fact, very few neonates from even alcoholic mothers present 'exencephaly' clinically, yet almost all of them will show poor behavioural capabilities as they grow older $^{25}$. This indicates that CNS development is impaired in those morphologically normal-appearing babies with prenatal alcohol exposure. To find out the alterations in such CNS tissues, we analysed the whole-brain protein changes of mice fetuses with prenatal alcohol exposure (without exencephaly, however) in the present investigation.

FA, a member of the B vitamin group, has been found to prevent birth defects, especially CNS abnormalities ${ }^{26-28}$. In the present study, we investigated changes of the fetal brain proteome, and found that some essential enzymes, which were modified by prenatal alcohol exposure, were protected by FA supplementation.

\section{Enzymes in metabolism and energy production}

Glucose is recognised as the key energy source for brain tissue; in the mean time, it also plays a role in the production of various important molecules, such as nucleic acids, lipids and certain neurotransmitters ${ }^{4}$. Physiologically, most glucose is oxidised by glycolysis to pyruvate and further oxidised into acetyl-CoA, which enters into the tricarboxylic acid cycle and produces more of the energy substance ATP under normoxic conditions. However, under hypoxic conditions, pyruvate will be reduced into lactate by lactate dehydrogenase, and no further ATP is produced in this reaction. Therefore far more ATP is produced by glucose oxidative phosphorylation than by anaerobic glycolysis. In the present investigation, the expression of two isocitrate dehydrogenases, which are key enzymes in the tricarboxylic acid cycle, was significantly down regulated in the fetal brain by prenatal alcohol exposure. This was very likely to impair the process of the tricarboxylic acid cycle and decrease the energy supply to the brain. On the other hand, lactate dehydrogenase, the enzyme catalysing the final step of anaerobic glycolysis, was up regulated by alcohol exposure. This was probably an adaptive response of the fetuses to the hypoxia caused by alcohol metabolism ${ }^{29}$.
As is mentioned earlier, under hypoxic conditions, ATP production through anaerobic glycolysis was limited, which might aggravate the energy insufficiency caused by down regulation of the two isocitrate dehydrogenases. Mitochondria play an essential role in ATP production, being where the tricarboxylic acid cycle takes place and most ATP molecules are produced. ATP synthesis through the mitochondrial respiratory chain is the fundamental means of cell energy production both in man and in animals. ATP synthase, also known as complex $\mathrm{V}$, is a key enzymic complex for ATP synthesis. In our previous study ${ }^{30}$, we found that in utero alcohol exposure could inhibit the activity of fetal brain ATP synthase. In the present investigation, it was found that the expression of two subunits of this complex ( $\alpha$ and D) was suppressed by alcohol, which would directly result in disturbed energy production in the developing brain. From the analysis above, the total energy supply in the fetal brain appeared to be decreased. Energy is crucial for developing organs. For the fetal brain, long-term energy insufficiency might cause retarded proliferation and differentiation of neurocytes and disturbed neurotransmitter formation. This might partly explain the microcephaly and mental retardation found in FAS patients.

\section{Signalling proteins}

Serine/threonine protein phosphatase, COP9 signalosome complex and nucleoside diphosphate kinase B are essential factors for signal transduction in cells and for the control mechanism of numerous physiological processes including growth, differentiation, metabolism, cell-cycle regulation and cytoskeleton function. 14-3-3 Protein could interact with a range of protein kinase, phosphatase and other signalling proteins, known as a type of 'adapter protein'. As well as interactions with proteins, including transcription factors, 14-3-3 isoforms have been shown to interact with cruciform $\mathrm{DNA}^{31}$, regulating the initiation of DNA replication. In the present investigation, prenatal alcohol exposure suppressed the expression of these signalling proteins in the fetal brain, which might result in certain developmental signalling pathway disturbance, thus contributing to CNS retardation or malformation. 


\section{Enzymes in protein translation and folding}

Proteasomes play an important role in cell growth and differentiation by removing denatured, damaged or improperly translated proteins from cells and regulating the level of proteins such as cyclins or some transcription factors. Changes in these enzymes are likely to result in delay or inaccuracy of protein translation and folding, and therefore affect the natural function of the proteins. In the present investigation, prenatal alcohol exposure suppressed the subunit expression of these enzymes in the fetal brain, which might be related to the structural and functional abnormalities of the CNS.

\section{Translationally controlled tumour protein}

Translationally controlled tumour protein (TCTP) was another up regulated protein by prenatal alcohol in the present research. TCTP is not a tumour- or tissue-specific protein, but is expressed ubiquitously from plants to mammals. It is believed to be important for cell growth and division. Overexpression of TCTP in mammalian cells results in slow growth and a delay in cell-cycle progression ${ }^{32}$. Overexpression of TCTP mutated in the phosphorylation sites for the mitotic kinase Plk disrupts the completion of mitosis ${ }^{33}$. Jung et al. ${ }^{34}$ reported recently that TCTP interacted with $\mathrm{Na}, \mathrm{K}-\mathrm{ATPase}$ and suppressed its activity. In the present investigation, prenatal alcohol exposure up regulated the expression of TCTP in the fetal brain, which might lead to a delay in cellcycle progression and slow growth of the fetal organ.

In addition to the effects described earlier, prenatal alcohol exposure also up regulated the level of $N^{G}, N^{G}$-dimethylarginine dimethylaminohydrolase 2 , an enzyme involved in $\mathrm{NO}$ generation. This enzyme hydrolyses $N^{G}, N^{G}$-dimethyl-L-arginine and $N^{G}$-monomethyl-L-arginine, which act as inhibitors of NO synthase, and therefore has a role in NO generation. In the present study, the NO synthase inhibition effect was likely to be reduced resulting from $N^{G}, N^{G}$-dimethylarginine dimethylaminohydrolase 2 up regulation, and therefore more NO would be produced. NO is well known as a highly diffusible, short-lived non-electrolyte chemical radical ${ }^{35}$. Although NO may have some physiological roles, excessive NO would exhibit pathophysiological effects. $\mathrm{NO}$ would interact with $\mathrm{O}_{2}$ to form nitrite $\left(\mathrm{NO}_{2}^{-}\right)$and nitrate $\left(\mathrm{NO}_{3}^{-}\right)$, or with the superoxide anion to form the highly reactive peroxynitrite $\left(\mathrm{ONOO}^{-}\right)$, which would lead to oxidative impairment of the developing brain tissue.

Altogether, under the dosage of the present investigation, FA supplementation mainly reversed the alcohol-altered proteins related to energy production, signalling and protein translation. However, there were still proteins altered by prenatal alcohol exposure yet not reversed by FA as narrated in the results. Most of those FA-protected proteins found in the present study really play essential roles in brain development. As a result, the fetal brain:body weight ratio of the FA supplementation group reversed to the control level. Those expression alterations caused by alcohol may have resulted from biogenesis inhibition of those enzymes at the transcription or translation level by alcohol, and/or induced by the damage by excess oxygenated free radicals generated by alcohol metabolism. 
FA is known to act as a cofactor for enzymes that are essential in DNA and RNA synthesis by providing single carbon units for the de novo synthesis of nucleotide bases. On the other hand, FA plays an important role in the transfer of methyl groups in the amino acid methylation cycle, an essential step in the recycling of homocysteine back to methionine. Because the requirement of nucleic acid and protein synthesis are at their peak during the stages of embryogenesis and rapid fetal growth, the demand for FA increases during pregnancy. However, alcohol ingestion inhibits FA absorption, which may lead to FA deficiency ${ }^{36-38}$. When FA deficiency reaches a certain degree, inhibition of the methylation cycle might impair the synthesis and function of specific proteins ${ }^{39-41}$. This may contribute to the decrease of some proteins' abundance found in the present research. However, when enough FA is supplemented, the organic nucleic acid and protein synthesis can return to the physiological level, and therefore the malformation and dysfunction caused by alcohol can be reversed. Still, it has been found that the metabolism process and metabolites of FA could counteract the oxidative stress ${ }^{42}$ and therefore reduce the oxidative impairments caused by alcohol. Additionally, FA may also play a role in the regulation of gene expression, which is known as gene-nutrient interaction $^{43}$, as was indicated in those studies of mutation and polymorphism screening of genes encoding proteins involved in FA metabolism and uptake.

What is also worth highlighting is that in our previous study with $\mathrm{CD}-1$ mice $^{44}$, we found that combinatorial supplementation of $\mathrm{FA}$ and vitamin $\mathrm{B}_{12}$ during the period of GD1-GD16 had a better protective effect against prenatal alcohol-induced fetal gross malformation and developmental retardation of the skeleton than FA alone. One explanation for the results is that $\mathrm{FA}$ and vitamin $\mathrm{B}_{12}$ are metabolically interdependent; therefore additional supplementation of vitamin $B_{12}$ may enhance the utilisation and biological effects of FA. Although the difference of animal models used in the two studies should be taken into consideration, these previous results hinted that the combination of FA and vitamin $B_{12}$ might also have a better protective effect on the fetal brain proteome than FA alone; this is under study by us now.

\section{Acknowledgements}

The present study was supported by a grant from the National Natural Sciences Foundations of the People's Republic of China (no. 30271364).

\section{References}

1. Jones KL \& Smith DW (1973) Recognition of the fetal alcohol syndrome in early infancy. Lancet ii, 999-1001.

2. Berman RF \& Hannigan JH (2000) Effects of prenatal alcohol exposure on the hippocampus: spatial behaviour, electrophysiology, and neuroanatomy. Hippocampus 10, 94-110.

3. Mattson SN, Schoenfeld AM \& Riley EP (2001) Teratogenic effects of alcohol on brain and behaviour. Alcohol Res Health 25, 185-191.
4. Goodlett CR \& Horn KH (2001) Mechanisms of alcoholinduced damage to the developing nervous system. Alcohol Res Health 25, 175-184.

5. Samson HH (1986) Microcephaly and fetal alcohol syndrome: human and animal studies. In Alcohol and Brain Development, pp. 167-183 [JR West, editor]. New York: Oxford University Press.

6. Streissguth AP, Aase JM, Clarren SK, Randels SP, LaDue RA \& Smith DF (1991) Fetal alcohol syndrome in adolescents and adults. JAMA 265, 1961-1967.

7. Centers for Disease Control and Prevention (CDC) (1995) Update: trends in fetal alcohol syndrome - United States, 1979-1993. MMWR Morb Mortal Wkly Rep 44, 249-251.

8. Roebuck TM, Mattson SN \& Riley EP (1999) Behavioral and psychosocial profiles of alcohol-exposed children. Alcohol Clin Exp Res 23, 1070-1076.

9. Kelly SJ, Day N \& Streissguth AP (2000) Effects of prenatal alcohol exposure on social behavior in humans and other species. Neurotoxicol Teratol 22, 143-149.

10. Sampson PD, Streissguth AP, Bookstein FL, Little RE, Clarren SK, Dehaene P, Hanson JW \& Graham JM Jr (1997) Incidence of fetal alcohol syndrome and prevalence of alcohol-related neurodevelopmental disorders. Teratology 56, 317-326.

11. Streissguth AP \& O'Malley K (2000) Neuropsychiatric implications and long-term consequences of fetal alcohol spectrum disorders. Semin Clin Neuropsychiatry 5, 177-190.

12. Kaneko WM, Riley EP \& Ehlers CL (1993) Electrophysiological and behavioral findings in rats prenatally exposed to alcohol. Alcohol 10, 169-178.

13. Cronise K, Marino MD, Tran TD \& Kelly SJ (2001) Critical periods for the effects of alcohol exposure on learning in rats. Behav Neurosci 115, 138-145.

14. Shah KR \& West M (1984) Behavioral changes in rat following perinatal exposure to ethanol. Neurosci Lett 47, 145-148.

15. Shaw GM, Lammer EJ, Wasserman CR, O'Malley CD \& Tolarova MM (1995) Risks of orofacial clefts in children born to women using multivitamins containing folic acid periconceptionally. Lancet 345, 393-396.

16. Kalter H (2000) Folic acid and human malformations: a summary and evaluation. Reprod Toxicol 14, 463-476.

17. Cogswell ME, Weisberg P \& Spong C (2003) Cigarette smoking, ethanol use and adverse pregnancy outcomes: implications for micronutrient supplementation. $J$ Nutr 133, 1722S-1731S.

18. Bailey LB, Rampersaud GC \& Kauwell GP (2003) Folic acid supplements and fortification affect the risk for neural tube defects, vascular disease and cancer: evolving science. $J$ Nutr 133, 1961S-1968S.

19. Lalonde R, Joyal CC \& Botez MI (1993) Effects of folic acid and folinic acid on cognitive and motor behaviors in 20-month-old rats. Pharm Biochem Behav 44, 703-707.

20. Kronick JN (1976) Teratogenic effects of ethyl ethanol administered to pregnant mice. Am J Obstet Gynecol 124, 676-680.

21. Essien FB \& Wannberg SL (1993) Methionine but not folinic acid or vitamin $B_{12}$ alters the frequency of neural tube defects in Axd mutant mice. J Nutr 123, 27-34.

22. Padmanabhan R, Ibrahim A \& Bener A (2002) Effect of maternal methionine pre-treatment on ethanol-induced exencephaly and axial skeletal dysmorphogenesis in mouse fetuses. Drug Alcohol Depend 65, 263-281.

23. Bradford M (1976) A rapid and sensitive method for the quantitation of microgram quantities of protein utilizing the principle of protein-dye binding. Anal Biochem 72, $248-254$.

24. Hannigan JH (1996) What research with animals is telling us about alcohol related neuro-developmental disorder. Pharmacol Biochem Behav 33, 489-499. 
25. Stoler JM \& Holmes LB (1999) Under-recognition of prenatal alcohol effects in infants of known alcohol abusing women. $J$ Pediatr 135, 430-436.

26. Anonymous (1991) Prevention of neural tube defects: results of the Medical Research Council vitamin study. MRC Vitamin Study Research Group. Lancet 338, 131-137.

27. Czeizel AE \& Dudas I (1992) Prevention of the first occurrence of neural tube defects by periconceptional vitamin supplementation. $N$ Engl J Med 327, 1832-1835.

28. Jae-Ho S \& Kohei S (1999) Folic acid supplementation of pregnant mice suppresses heat-induced neural tube defects in the offspring. J Nutr 129, 2070-2073.

29. Guerri C (1998) Neuroanatomical and neurophysiological mechanisms involved in central nervous system dysfunctions induced by prenatal alcohol exposure. Alcohol Clin Exp Res 22, 304-312.

30. Xu Y, Liu P \& Li Y (2005) Impaired development of mitochondria plays a role in the fetal alcohol syndrome. Birth Defects Res A 73, 83-91.

31. Todd A, Cossons N, Aitken A, Price GB \& Zannis-Hadjopoulos M (1998) Human cruciform binding protein belongs to the 14-33 family. Biochemistry 37, 14317-14325.

32. Gachet Y, Tournier S, Lee M, Lazaris-Karatzas A, Poulton T \& Bommer UA (1999) The growth-related, translationally controlled protein $\mathrm{P} 23$ has properties of a tubulin binding protein and associates transiently with microtubules during the cell cycle. J Cell Sci 112, 1257-1271.

33. Yarm FR (2002) Plk phosphorylation regulates the microtubulestabilizing protein TCTP. Mol Cell Biol 22, 6209-6221.

34. Jung J, Kim M, Kim MJ, Kim J, Moon J, Lim JS, Kim M \& Lee K (2004) Translationally controlled tumor protein interacts with the third cytoplasmic domain of $\mathrm{Na}, \mathrm{K}$-ATPase $\alpha$ subunit and inhibits the pump activity in HeLa cells. $J$ Biol Chem $\mathbf{2 7 9}$, 49868-49875.

35. Davis RL \& Syapin PJ (2005) Interactions of alcohol and nitricoxide synthase in the brain. Brain Res Brain Res Rev 49 , 494-504.

36. McGuffin R, Goff P \& Holman RS (1975) Effect of diet and ethanol on the development of folate deficiency in the rat. $\mathrm{Br}$ J Haematol 31, 185-192.

37. McMartin KE, Shiao CQ, Collins TD \& Redetzki HM (1985) Acute ethanol ingestion by humans and subacute treatment of rats increase urinary folate excretion. Alcohol 2, 473-477.

38. Muldoon RT \& McMartin KE (1994) Ethanol acutely impairs the renal conservation of 5-methyltetrahydrofolate in the isolated perfused rat kidney. Ethanol Clin Exp Res 18, 333-339.

39. Wagner C (1995) Biochemical role of folate in cellular metabolism. In Folate in Health and Disease, pp. 23-42 [LB Bailey, editor]. New York: Marcel Dekker Inc.

40. Hibbard BM (1964) The role of folic acid in pregnancy; with particular reference to anaemia, abruption and abortion. $J$ Obstet Gynecol Br Commonw 71, 529-542.

41. Chanarin I, Rothman D, Ward A \& Perry J (1968) Folate status and requirement in pregnancy. Br Med J 2, 390-394.

42. Verhaar MC, Stores E \& Rabelink TJ (2002) Folic acids and cardiovascular disease. Arterioscler Thromb Vasc Biol 22, 6-13.

43. Finnell RH, Shaw GM, Lammer EJ, Brandl KL, Carmichael SL \& Rosenquist TH (2004) Gene-nutrient interactions: importance of folates and retinoids during early embryogenesis. Toxicol Appl Pharm 198, 75-85.

44. Xu Y, Li Y, Tang Y, Wang K, Shen X, Long Z \& Zheng X (2006) The maternal combined supplementation of folic acid and vitamin $\mathrm{B}(12)$ suppresses ethanol-induced developmental toxicity in mouse fetuses. Reprod Toxicol 22, 56-61. 